IMA Journal of Applied Mathematics (2007) 72, 659-679

doi:10.1093/imamat/hxm033

Advance Access publication on September 26, 2007

\title{
Existence and stability of TE modes in a stratified non-linear dielectric
}

\author{
C. A. STUART $\dagger$ \\ Station 8, Institut d'Analyse et Calcul Scientifique-Faculté de Sciences de Base, \\ EPFL, CH-1015 Lausanne, Switzerland
}

[Received on 1 September 2006; accepted on 29 January 2007]

\begin{abstract}
Starting from Maxwell's equations for a stratified optical medium with a non-linear refractive index, we derive the equations for monochromatic planar TE modes. It is then shown that TE modes in which the electromagnetic fields are travelling waves correspond to solutions of these reduced equations in the form of standing waves. The equations of the paraxial approximation are then formulated and the stability of the travelling waves is investigated in that context.
\end{abstract}

Keywords: non-linear waveguide; TE modes; travelling waves; orbital stability.

\section{Introduction}

We consider the propagation of monochromatic light through a stratified non-linear dielectric. The ability of such a medium to support guided waves depends both on the spatial variation of the refractive index across the layers and on its non-linear response to the intensity of the beam of light. The mathematical model for this involves studying special solutions of Maxwell's equations together with a nonlinear constitutive assumption. In Stuart (1993), the basic equations were derived and a variety of results were proved concerning the existence, non-existence and dependence on the physical parameters of such solutions. See Ruppen (1997) and Arcoya et al. (1999) for further work on the mathematical theory. We are now able to continue the study of this problem in some new directions.

First of all, in Section 3, we derive the equations governing all planar monochromatic solutions of Maxwell's equations in a non-linear optical medium. These equations form a system of six coupled second-order partial differential equations in the plane, see (3.13). TE and TM modes are special solutions of this system in which some of the components of the solutions are identically zero. In Section 5, we introduce the notion of guided waves. These are solutions satisfying additional conditions ensuring that the energy is finite and that the fields decay to zero far from the centre of the waveguide. Among all planar monochromatic solutions of Maxwell's equation, those corresponding to travelling waves are of particular interest and for them the system reduces to six second-order ordinary differential equations. Travelling waves are introduced in Section 4. The discussion in Stuart (1993), Ruppen (1997) and Arcoya et al. (1999), which deals exclusively with guided TE modes that are planar travelling waves, can now be seen as part of a broader context. This is an essential preliminary step in order to formulate in a precise way issues concerning the stability of guided TE travelling waves. So far there seems to have been no progress on establishing stability in the context of Maxwell's equations. Instead, stability is usually discussed with respect to a non-linear Schrödinger equation (NLS) that is obtained from the paraxial approximation. In Section 6, we show how such an equation is derived in our setting. The stability of guided TE travelling waves is then shown to correspond to the orbital stability of certain

\footnotetext{
${ }^{\dagger}$ Email: charles.stuart@epfl.ch
} 
periodic solutions (standing waves) of this NLS. However, since all perturbed fields admitted in this treatment are monochromatic, it must be emphasized that the variable playing the role of time in the NLS is actually distance in the direction of propagation of the travelling wave whose stability is being analysed.

It is intuitively clear from Snell's law that a favorable configuration for the existence of guided waves is found in a wave guide in which the refractive index decreases away from some central axis. It is also well-known that the guidance properties are enhanced by the non-linear response of a self-focusing medium. In Section 7, using work from Jeanjean \& Stuart (1999) and McLeod et al. (2003), we present a fairly complete picture of the existence and stability of guided TE travelling waves in such a structure. Under appropriate conditions, all the ground states lie on a smooth branch and they are all orbitally stable.

\section{Maxwell's equations in a non-linear dielectric}

In this section, we review briefly Maxwell's equations for electromagnetic waves in a non-linear dielectric which is supposed to occupy all of $\mathbb{R}^{3}$. Let $E$ and $B: \mathbb{R}^{4} \rightarrow \mathbb{R}^{3}$ denote the electric and magnetic fields and let $P: \mathbb{R}^{4} \rightarrow \mathbb{R}$ denote the polarization of the medium due to the presence of these fields. In CGS units, Maxwell's equations are

$$
\begin{aligned}
& \partial_{t} B=-c \nabla \wedge E, \text { (1) } \partial_{t} E=c \nabla \wedge B-\partial_{t} P, \\
& \nabla \cdot B=0, \quad \text { (3) } \nabla \cdot E=-\nabla \cdot P
\end{aligned}
$$

where $c>0$ is speed of light in a vacuum. On the microscopic level, the atoms and molecules of the dielectric are polarized into dipoles by the electric field and $P$ is the macroscopic manifestation of this phenomenon. In a given material, the way in which $P$ is determined by $E$ must be specified by a constitutive relation. In the context of transparent isotropic optical waveguides, this response is postulated to have the form

$$
P(x, t)=\chi\left(\omega, x, \frac{1}{2}\left[\left|E^{1}(x)\right|^{2}+\left|E^{2}(x)\right|^{2}\right]\right) E(x, t) ;
$$

for a monochromatic electric field of frequency $\omega>0$,

$$
E(x, t)=E^{1}(x) \cos \omega t+E^{2}(x) \sin \omega t,
$$

where $E^{1}, E^{2}: \mathbb{R}^{3} \rightarrow \mathbb{R}^{3}$. The function $\chi:(a, b) \times \mathbb{R}^{3} \times[0, \infty) \rightarrow(0, \infty)$ is called the dielectric susceptibility', for some interval of frequencies $(a, b)$. The fact that $P$ is also monochromatic with the same frequency as $E$ (and so second and third harmonics, etc., are neglected) is appropriate for situations in which there is no phase matching (see Saleh $\&$ Teich, 1991, e.g.). Furthermore, $\chi$ depends on $E$ only through $\frac{1}{2}\left[\left|E^{1}(x)\right|^{2}+\left|E^{2}(x)\right|^{2}\right]$ which is the time average of $|E(x, t)|^{2}$.

It is customary to introduce the 'electric displacement field' $D$ by setting

$$
D=E+P \text {. }
$$

Maxwell's equations in a dielectric can then be written as

$$
\begin{aligned}
& \partial_{t} B=-c \nabla \wedge E, \text { (1) } \partial_{t} D=c \nabla \wedge B, \\
& \nabla \cdot B=0, \quad \text { (3) } \nabla \cdot D=0,
\end{aligned}
$$


and the 'constitutive relation' is

$$
D(x, t)=\left\{1+\chi\left(\omega, x, \frac{1}{2}\left[\left|E^{1}(x)\right|^{2}+\left|E^{2}(x)\right|^{2}\right]\right)\right\} E(x, t),
$$

where the function

$$
\varepsilon(\omega, x, s)=1+\chi(\omega, x, s)
$$

is known as the 'dielectric response'.

The quantity $n(\omega, x, s)=\sqrt{\varepsilon(\omega, x, s)}$ is usually referred to the 'non-linear refractive index'. It is a function of position $x$, but depends also on the monochromatic electric field $E(x, t)$ through its frequency $\omega$ and intensity $s$. To a first approximation, the refractive index can be written as

$$
n(\omega, x, s)=n_{0}(\omega, x)+n_{2}(\omega, x) s,
$$

and this is usually referred to as a 'Kerr non-linearity' (Akhmanov et al., 1972; Svelto, 1974). There are extensive tables for the coefficients $n_{0}$ and $n_{2}$ (Smith, 1986). See also Kivshar \& Sukhorukov (2001), Luther-Davies \& Stegeman (2001) and the references in Stuart (1993) for a discussion of more general non-linearities. A medium is 'self-focusing' when $\varepsilon(\omega, x, s)$ is an increasing function of $s$.

By (2.5), $\partial_{t} \frac{1}{2}\left\{E \cdot D+|B|^{2}\right\}=\partial_{t} D \cdot E+\partial_{t} B \cdot B$ and so, for any smooth bounded domain $V$ in $\mathbb{R}^{3}$,

$$
\partial_{t} \int_{V} \frac{1}{2}\left\{E \cdot D+|B|^{2}\right\} \mathrm{d} x=c \int_{V}\{\nabla \wedge B \cdot E-\nabla \wedge E \cdot B\} \mathrm{d} x=-c \int_{\partial V} E \wedge B \cdot n \mathrm{~d} s,
$$

where $n: \partial V \rightarrow \mathbb{R}^{3}$ is the field of unit exterior normals on $\partial V$. The quantity $\frac{1}{2}\left\{E \cdot D+|B|^{2}\right\}$ is considered to be the 'electromagnetic energy density'. For any smooth orientable surface $S$ in $\mathbb{R}^{3}$,

$$
c \int_{S}(E \wedge B) \cdot n \mathrm{~d} s
$$

is the rate at which this energy is flowing across $S$ in the direction of a continuous field $n: S \rightarrow \mathbb{R}^{3}$ of unit normals. Note that, unlike the energy density, this 'energy flux' is independent of the polarization $P$.

\section{Planar monochromatic solutions of Maxwell's equations}

Let $x=\left(x_{1}, x_{2}, x_{3}\right) \in \mathbb{R}^{3}$ denote Cartesian co-ordinates in space and let $t \in \mathbb{R}$ denote time. As in Section 2 , a field $F: \mathbb{R}^{4} \rightarrow \mathbb{R}^{3}$ is 'monochromatic' if

$$
F(x, t)=F^{1}(x) \cos \omega t+F^{2}(x) \sin \omega t, \quad \text { for } x \in \mathbb{R}^{3} \text { and } t \in \mathbb{R},
$$

for some 'frequency' $\omega>0$ and functions $F^{1}, F^{2}: \mathbb{R}^{3} \rightarrow \mathbb{R}^{3}$. For such fields, the 'time average' of $|F(x, t)|^{2}$ is $\frac{1}{2}\left\{\left|F^{1}(x)\right|^{2}+\left|F^{2}(x)\right|^{2}\right\}$ and this function will be referred to as the 'intensity distribution' of the field $F$.

A field $F: \mathbb{R}^{4} \rightarrow \mathbb{R}^{3}$ is 'planar' with respect to a direction $\eta \in \mathbb{R}^{3}$ if

$$
F(x+s \eta, t)=F(x, t), \quad \text { for } x \in \mathbb{R}^{3} \text { and } s, t \in \mathbb{R} .
$$

Henceforth, we when dealing with planar fields we shall use a Cartesian co-ordinate system such that the fields are planar with respect to the $x_{2}$-axis. Then, we simplify the notation by using

$$
\left(x_{1}, x_{2}, x_{3}\right)=(x, y, z) \text { and } F(x, y, z, t)=F^{1}(x, z) \cos \omega t+F^{2}(x, z) \sin \omega t
$$

to denote a planar monochromatic field where now $F^{1}, F^{2}: \mathbb{R}^{2} \rightarrow \mathbb{R}^{3}$. 
When dealing with such fields, it is convenient to use complex notation since

$$
F^{1}(x, z) \cos \omega t+F^{2}(x, z) \sin \omega t=\operatorname{Re}\left\{F(x, z) \mathrm{e}^{-\mathrm{i} \omega t}\right\},
$$

where $F(x, z)=F^{1}(x, z)+\mathrm{i} F^{2}(x, z) \in \mathbb{C}^{3}$.

As a model for a 'stratified medium', we assume henceforth that

$$
\varepsilon(\omega, x, y, z, s)=\mu(\omega, x, s)
$$

for some function $\mu:(a, b) \times \mathbb{R} \times[0, \infty) \rightarrow \mathbb{R}$, where for $\omega \in(a, b) \subset(0, \infty)$,

(i) $\mu(\omega, \cdot, \cdot) \in C^{1}(\mathbb{R} \times[0, \infty))$ and, for all $K>0$,

$\mu(\omega, \cdot, \cdot)$ is bounded and uniformly continuous on $\mathbb{R} \times[0, K]$.

(ii) $\inf \{\mu(\omega, x, s): x \in \mathbb{R}$ and $s \geqslant 0\}>0$.

From now on, we look for solutions of Maxwell's equations in which the electric field is planar and monochromatic. Thus, it can be expressed in the form

$$
E(x, y, z, t)=\operatorname{Re}\left\{e(x, z) \mathrm{e}^{-\mathrm{i} \omega t}\right\}, \quad \text { where } e(x, z)=e^{1}(x, z)+\mathrm{i} e^{2}(x, z),
$$

where $e: \mathbb{R}^{2} \rightarrow \mathbb{C}^{3}$. The intensity distribution of such a field is

$$
\frac{1}{2}\left[\left|e^{1}(x, z)\right|^{2}+\left|e^{2}(x, z)\right|^{2}\right]=\frac{1}{2}|e(x, z)|^{2}=\frac{1}{2} \sum_{j=1}^{2} \sum_{m=1}^{3} e_{m}^{j}(x, z)^{2},
$$

and so, by (2.5), the associated displacement field is

$$
D(x, y, z, t)=\mu\left(\omega, x, \frac{1}{2}|e(x, z)|^{2}\right) \operatorname{Re}\left\{e(x, z) \mathrm{e}^{-\mathrm{i} \omega t}\right\} .
$$

From the equation $\partial_{t} B=-c \nabla \wedge E$, we find that

$$
B(x, y, z, t)-B(x, y, z, 0)=-c \int_{0}^{t} \nabla \wedge E(x, y, z, s) \mathrm{d} s=c \operatorname{Re}\left\{\nabla \wedge e(x, z) \frac{1}{\mathrm{i} \omega}\left[\mathrm{e}^{-\mathrm{i} \omega t}-1\right]\right\},
$$

and so

$$
B(x, y, z, t)=-\frac{c}{\omega} \operatorname{Re}\left\{\mathrm{i} \nabla \wedge e(x, z) \mathrm{e}^{-\mathrm{i} \omega t}\right\}
$$

provided that we choose the initial condition $B(x, y, z, 0)=-\frac{c}{\omega} \operatorname{Re}\{\mathrm{i} \nabla \wedge e(x, z)\}$. Thus, $B$ and $D$ are also planar monochromatic fields with

$$
B(x, y, z, t)=\operatorname{Re}\left\{b(x, z) \mathrm{e}^{-\mathrm{i} \omega t}\right\} \quad \text { and } \quad D(x, y, z, t)=\operatorname{Re}\left\{d(x, z) \mathrm{e}^{-\mathrm{i} \omega t}\right\},
$$

where

$$
b(x, z)=-\mathrm{i} \frac{c}{\omega} \nabla \wedge e(x, z) \quad \text { and } \quad d(x, z)=\mu\left(\omega, x, \frac{1}{2}|e(x, z)|^{2}\right) e(x, z) .
$$


Maxwell's equation $\partial_{t} D=c \nabla \wedge B$ becomes

$$
\mu\left(\omega, x, \frac{1}{2}|e(x, z)|^{2}\right) \operatorname{Re}\left\{\mathrm{i} \omega \mathrm{e}^{-\mathrm{i} \omega t}\right\}=\frac{c^{2}}{\omega} \operatorname{Re}\left\{\mathrm{i} \nabla \wedge \nabla \wedge e(x, z) \mathrm{e}^{-\mathrm{i} \omega t}\right\} .
$$

This equation is satisfied for all $t \in \mathbb{R}$, if and only if $e(x, z)$ satisfies the equation

$$
\mu\left(\omega, x, \frac{1}{2}|e(x, z)|^{2}\right) e(x, z)=\left(\frac{c}{\omega}\right)^{2} \nabla \wedge \nabla \wedge e(x, z)=\left(\frac{c}{\omega}\right)^{2}\{\nabla(\nabla \cdot e(x, z))-\Delta e(x, z)\},
$$

which is actually a system of three equations for $e: \mathbb{R}^{2} \rightarrow \mathbb{C}^{3}$, namely

$$
\begin{array}{r}
\left(\frac{\omega}{c}\right)^{2} \mu\left(\omega, x, \frac{1}{2}|e(x, z)|^{2}\right)\left(\begin{array}{l}
e_{1} \\
e_{2} \\
e_{3}
\end{array}\right)=\left(\begin{array}{c}
\partial_{z x}^{2} e_{3}-\partial_{z z}^{2} e_{1} \\
-\partial_{x x}^{2} e_{2}-\partial_{z z}^{2} e_{2} \\
\partial_{z x}^{2} e_{1}-\partial_{x x}^{2} e_{3}
\end{array}\right) \\
\text { where } e_{m}=e_{m}(x, z), \text { for } m=1,2,3 .
\end{array}
$$

Thus, we see that for planar monochromatic fields, Maxwell's equation reduce to the system (3.13). The following more precise statement can be checked by straightforward calculations.

THEOREM 3.1 Let the dielectric response function $\mu$ satisfy (3.4)-(3.6) and let $e \in C^{2}\left(\mathbb{R}^{2}, \mathbb{C}^{3}\right)$ satisfy the system (3.13). Define $E, D$ and $B$ by (3.7), (3.11) and (3.12). Then, $E, B, D$ satisfy Maxwell's equations (2.4).

We observe that the equations of the system (3.13) are coupled through the dielectric response

$$
\mu\left(\omega, x, \frac{1}{2}|e(x, z)|^{2}\right)=\mu\left(\omega, x, \frac{1}{2} \sum_{j=1}^{2} \sum_{m=1}^{3} e_{m}^{j}(x, z)^{2}\right) .
$$

However, it is possible to seek special solutions in which some of the components of $e(x, z)$ are identically zero.

\subsection{TE modes}

The simplest kind of solution is one in which $e_{1} \equiv e_{3} \equiv 0$, in which case the system (3.13) reduces to a single equation for the complex function $e_{2}=e_{2}^{1}+\mathrm{i} e_{2}^{2}$, namely

$$
\left(\frac{\omega}{c}\right)^{2} \mu\left(\omega, x, \frac{1}{2}\left|e_{2}(x, z)\right|^{2}\right) e_{2}=-\partial_{x x}^{2} e_{2}-\partial_{z z}^{2} e_{2} .
$$

These solutions are referred to as 'TE modes' since the electric field $E$ is everywhere transverse to the direction of the $z$-axis.

\subsection{TM modes}

Another, somewhat more complicated, type of solution is obtained by requiring that $e_{2} \equiv 0$. Then, we are left with a $2 \times 2$ system for the complex functions $e_{1}$ and $e_{3}$, namely

$$
\left(\frac{\omega}{c}\right)^{2} \mu\left(\omega, x, \frac{1}{2}\left[\left|e_{1}(x, z)\right|^{2}+\left|e_{3}(x, z)\right|^{2}\right]\right)\left(\begin{array}{c}
e_{1} \\
e_{3}
\end{array}\right)=\left(\begin{array}{c}
\partial_{z x}^{2} e_{3}-\partial_{z z}^{2} e_{1} \\
\partial_{z x}^{2} e_{1}-\partial_{x x}^{2} e_{3}
\end{array}\right) .
$$


Note that if $e=\left(e_{1}, 0, e_{3}\right)$ is such a solution, then the corresponding magnetic field defined by (3.10) has the property that $B_{3}(x, y, z, t) \equiv 0$ and so solutions of this type are referred to as 'TM modes' since the magnetic field is everywhere transverse to the direction of the $z$-axis.

\section{Monochromatic planar travelling waves}

A field $F: \mathbb{R}^{4} \rightarrow \mathbb{R}^{3}$ is called a 'travelling wave' if

$$
F(x, t)=w(x-t \xi), \quad \text { for } x \in \mathbb{R}^{3} \text { and } t \in \mathbb{R},
$$

for some vector $\xi \in \mathbb{R}^{3} \backslash\{0\}$ and function $w: \mathbb{R}^{3} \rightarrow \mathbb{R}^{3}$. In this case, $\frac{\xi}{|\xi|}$ is a 'direction of propagation' and $v=|\xi|$ is the 'wave speed' in this direction.

LEMMA 4.1 Let $F: \mathbb{R}^{4} \rightarrow \mathbb{R}^{3}$ be a monochromatic field with frequency $\omega>0$ that is also a travelling wave in the direction $(0,0,1)$ with speed $v>0$.Then, there exist fields $K^{1}, K^{2}: \mathbb{R}^{2} \rightarrow \mathbb{R}^{3}$ such that

$$
F(x, t)=K^{1}\left(x_{1}, x_{2}\right) \cos \left(k x_{3}-\omega t\right)+K^{2}\left(x_{1}, x_{2}\right) \sin \left(k x_{3}-\omega t\right),
$$

for all $(x, t) \in \mathbb{R}^{4}$, where $k=\frac{\omega}{v}$ is the 'wave number' and $\frac{2 \pi}{k}$ is the 'wave length'.

Proof. See Lemma 2.1 of Stuart (2004).

By this lemma and the discussion at the beginning of Section 3, monochromatic planar fields that are travelling waves in the direction of the $z$-axis are of the form

$$
F(x, y, z, t)=K^{1}(x) \cos (k z-\omega t)+K^{2}(x) \sin (k z-\omega t)=\operatorname{Re} K(x) \mathrm{e}^{\mathrm{i}(k z-\omega t)},
$$

where $K^{1}, K^{2}: \mathbb{R} \rightarrow \mathbb{R}^{3}$ and $K=K^{1}-\mathrm{i} K^{2}$.

Consequently, solutions $e(x, z)$ of the system (3.13) yield travelling waves propagating in the direction of the $z$-axis if and only if there exist a constant $k>0$ and a function $W: \mathbb{R} \rightarrow \mathbb{C}^{3}$ such that

$$
e(x, z)=W(x) \mathrm{e}^{\mathrm{i} k z}, \quad \text { for all } x, z \in \mathbb{R} .
$$

Then, the electric field has the form

$$
E(x, y, z, t)=\operatorname{Re}\left\{W(x) \mathrm{e}^{\mathrm{i}(k z-\omega t)}\right\},
$$

and Theorem 3.1 now yields the following result.

THEOREM 4.1 Let the dielectric response function $\mu$ satisfy (3.4)-(3.6) and let $k>0$ and $W \in C^{2}$ $\left(\mathbb{R}, \mathbb{C}^{3}\right)$ satisfy the system

$$
\left(\frac{\omega}{c}\right)^{2} \mu\left(\omega, x, \frac{1}{2}|W(x)|^{2}\right)\left(\begin{array}{c}
W_{1} \\
W_{2} \\
W_{3}
\end{array}\right)=\left(\begin{array}{c}
\mathrm{i} k \partial_{x} W_{3}+k^{2} W_{1} \\
-\partial_{x x}^{2} W_{2}+k^{2} W_{2} \\
i k \partial_{x} W_{1}-\partial_{x x}^{2} W_{3}
\end{array}\right),
$$

where $W_{m}=W_{m}(x)$, for $m=1,2,3$.

Set $e(x, z)=W(x) \mathrm{e}^{\mathrm{i} k z}$ and define $E, D$ and $B$ by (3.7), (3.11) and (3.12), respectively. Then, $E, B$ and $D$ satisfy Maxwell's equations (2.4) and they are planar monochromatic travelling waves propagating in the direction of the $z$-axis with speed $\frac{\omega}{k}$. 
Note that for travelling waves, the formula (3.10) simplifies to

$$
B(x, y, z, t)=\frac{c}{\omega} \operatorname{Re}\left\{\left(\begin{array}{c}
-k W_{2}(x) \\
k W_{1}(x)+\mathrm{i} \partial_{x} W_{3}(x) \\
-\mathrm{i} \partial_{x} W_{2}(x)
\end{array}\right) \mathrm{e}^{\mathrm{i}(k z-\omega t)}\right\} .
$$

Since $W(x)=W^{1}(x)+\mathrm{i} W^{2}(x)$, the system (4.6) constitutes a system of six coupled second-order differential equations for the real functions $W_{m}^{j} \in C^{2}(\mathbb{R})$, for $j=1,2$ and $m=1,2,3$.

The simplest solutions of (4.6) are those in which the function $W$ is constant. The electromagnetic fields are then plane waves propagating in a homogeneous medium

\subsection{Plane waves}

Suppose that, in addition to (3.4) and (3.6), the dielectric response function $\mu(\omega, x, s)$ is independent of $x$. This means that the medium is homogeneous and we write the response function simply as $\mu(\omega, s)$. Then, a constant vector $W \in \mathbb{C}^{3}$ satisfies (4.6) if and only if $W_{3}=0$ and

$$
\left(\frac{\omega}{c}\right)^{2} \mu\left(\omega, \frac{1}{2}|W|^{2}\right)\left(\begin{array}{l}
W_{1} \\
W_{2}
\end{array}\right)=\left(\begin{array}{l}
k^{2} W_{1} \\
k^{2} W_{2}
\end{array}\right) .
$$

This is possible with $W \not \equiv 0$ if and only if

$$
k=\frac{\omega}{c} \sqrt{\mu(\omega, s)}, \quad \text { for some } s>0 \text { and }|W|=\sqrt{2 s} .
$$

The electric and magnetic fields are then

$$
E=\operatorname{Re}\left\{\left(\begin{array}{c}
W_{1} \\
W_{2} \\
0
\end{array}\right) \mathrm{e}^{\mathrm{i}(k z-\omega t)}\right\} \text { and } B=\frac{c}{\omega} \operatorname{Re}\left\{\left(\begin{array}{c}
-k W_{2} \\
k W_{1} \\
0
\end{array}\right) \mathrm{e}^{\mathrm{i}(k z-\omega t)}\right\} .
$$

Note that for such solutions, which are called 'plane waves', $E, B$ and the $z$-axis are always mutually perpendicular.

If the medium is homogeneous and linear, the dielectric response is a constant depending only on $\omega$ and so the condition (4.8) becomes

$$
k=\frac{\omega}{c} \sqrt{\mu(\omega)} \text {, i.e. } \frac{\omega}{k}=\frac{c}{n(\omega)},
$$

and there is no restriction on the norm of $W$. The condition (4.9) is called the 'dispersion relation' and it determines the spatial wave length $\lambda=\frac{2 \pi}{k}$ of the plane waves as a function of the temporal frequency $\omega$. It also shows that the wave speed $\frac{\omega}{k}$ is determined by the refractive index in a linear homogeneous medium. In a non-linear medium, (4.8) shows that the wave speed $\frac{\omega}{k}$ depends on the intensity as well as the frequency through $\frac{\omega}{k}=\frac{c}{n(\omega, s)}$.

\section{Guidance conditions}

In Section 4, we showed that all planar, monochromatic travelling waves are obtained as solutions of a system of six second-order differential equations on $\mathbb{R}$. However, not all such solutions correspond 
to physically realistic and interesting situations. On one hand, acceptable solutions should have finite energy. Furthermore, the intensity of the associated beam of light should be concentrated near the axis of propagation. For planar waveguides, these additional criteria are formulated as follows.

\section{Guidance conditions}

(i) The total electromagnetic energy per unit length in the direction of propagation is finite. That is,

$$
\int_{d}^{d+1} \int_{-\infty}^{\infty} \frac{1}{2}\left\{E \cdot D+|B|^{2}\right\} \mathrm{d} x \mathrm{~d} z<\infty \quad \text { for all } d \in \mathbb{R} .
$$

(ii) The amplitudes of the electromagnetic fields decay to zero as the distance from the axis of propagation becomes infinite. That is,

$$
|E(x, y, z, t)| \rightarrow 0 \text { and }|B(x, y, z, t)| \rightarrow 0 \text { as }|x| \rightarrow \infty .
$$

For fields of the form (3.7), (3.11) and (3.12), the condition (i) is satisfied if and only if

$$
\int_{d}^{d+1} \int_{-\infty}^{\infty} \mu\left(\omega, x, \frac{1}{2}|e(x, z)|^{2}\right)|e(x, z)|^{2}+|\nabla \wedge e(x, z)|^{2} \mathrm{~d} x \mathrm{~d} z<\infty
$$

and the condition (ii) amounts to

$$
|e(x, z)| \rightarrow 0 \text { and }|\nabla \wedge e(x, z)| \rightarrow 0 \text { as }|x| \rightarrow \infty .
$$

In the case of travelling waves, $e(x, z)=W(x) \mathrm{e}^{\mathrm{i} k z}$ and these conditions become

$$
\int_{-\infty}^{\infty}\left[1+\mu\left(\omega, x, \frac{1}{2}|W(x)|^{2}\right)\right]|W(x)|^{2}+\left|\partial_{x} W_{2}\right|^{2}+\left|\partial_{x} W_{3}\right|^{2} \mathrm{~d} x<\infty
$$

and

$$
|W(x)|,\left|\partial_{x} W_{2}\right|,\left|\partial_{x} W_{3}\right| \rightarrow 0 \quad \text { as }|x| \rightarrow \infty
$$

respectively.

Clearly, plane waves do not satisfy the guidance conditions.

\subsection{Guided TE modes}

Recall that TE modes are solutions of (3.13) in which $e_{3}^{1} \equiv e_{3}^{2} \equiv 0$. Thus, we find TE modes by solving the non-linear Helmholtz equation

$$
\Delta w(x, z)+\left(\frac{\omega}{c}\right)^{2} \mu\left(\omega, x, \frac{1}{2}|w(x, z)|^{2}\right) w(x, z)=0, \quad \text { for }(x, z) \in \mathbb{R}^{2},
$$

for $w \in C^{2}\left(\mathbb{R}^{2}, \mathbb{C}\right)$ and then setting

$$
E=\operatorname{Re}\left\{\left(\begin{array}{c}
0 \\
w(x, z) \mathrm{e}^{-\mathrm{i} \omega t} \\
0
\end{array}\right)\right\} \quad \text { and } \quad B=\frac{c}{\omega} \operatorname{Re}\left\{\mathrm{i}\left(\begin{array}{c}
\partial_{z} w(x, z) \\
0 \\
-\partial_{x} w(x, z)
\end{array}\right) \mathrm{e}^{-\mathrm{i} \omega t}\right\}
$$


Under the assumptions (3.4)-(3.6), the guidance conditions (5.1) and (5.2) become

(i) $w \in H^{1}(\mathbb{R} \times(d, d+1))$, for all $d \in \mathbb{R}$, and

(ii) $|w(x, z)|$ and $|\nabla w(x, z)| \rightarrow 0$ as $|x| \rightarrow \infty$, for all $z \in \mathbb{R}$.

Note that for TE modes, the Poynting vector $E \wedge B$ is always in the direction of the $z$-axis, and, according to (2.7), the rate at which energy is crossing the line $z=d$ is

$$
c \int_{-\infty}^{\infty} E \wedge B \cdot e_{3} \mathrm{~d} x
$$

Using (5.6), the time average of this quantity is

$$
P(d)=\frac{c^{2}}{2 \omega} \int_{-\infty}^{\infty} \operatorname{Im}\left\{\overline{w(x, d)} \partial_{z} w(x, d)\right\} \mathrm{d} x .
$$

Proposition 5.1 Suppose that $\mu$ satisfies (3.4)-(3.6). If $w \in C^{2}\left(\mathbb{R}^{2}, \mathbb{C}\right)$ satisfies (5.5), (5.7) and (5.8), the quantity $P$ defined by (5.9) is finite and it is independent of $d$. It is called the 'power' of the corresponding TE mode.

Proof. By (5.7), $w \in H^{1}(\mathbb{R} \times(d, D))$ for all $d, D$ with $d<D$. In particular, $\partial_{z} w(x, z) \overline{w(x, z)} \in L^{1}(\mathbb{R})$ for almost all $z \in \mathbb{R}$, so we can choose $d$ such that $\partial_{z} w(x, d) \overline{w(x, d)} \in L^{1}(\mathbb{R})$. Furthermore, $|w||\nabla w| \in$ $L^{1}(\mathbb{R} \times(d, D))$ and so there exists a sequence $\left\{r_{n}\right\}$ such that $r_{n} \rightarrow \infty$ and

$$
\int_{d}^{D}\left|w\left(r_{n}, z\right)\right|\left|\nabla w\left(r_{n}, z\right)\right|+\left|w\left(-r_{n}, z\right)\right|\left|\nabla w\left(-r_{n}, z\right)\right| \mathrm{d} z \rightarrow \infty \quad \text { as } n \rightarrow \infty .
$$

Multiplying (5.5) by $\overline{w(x, z)}$ and then integrating over the rectangle $\left(-r_{n}, r_{n}\right) \times(d, D)$ yields

$$
\begin{aligned}
\int_{d}^{D} & \int_{-r_{n}}^{r_{n}}\left(\frac{\omega}{c}\right)^{2} \mu\left(\omega, x, \frac{1}{2}|w(x, z)|^{2}\right)|w(x, z)|^{2} \mathrm{~d} x \mathrm{~d} z=-\int_{d}^{D} \int_{-r_{n}}^{r_{n}} \Delta w(x, z) \overline{w(x, z)} \mathrm{d} x \mathrm{~d} z \\
= & \int_{d}^{D} \int_{-r_{n}}^{r_{n}}\left|\partial_{x} w\right|^{2}+\left|\partial_{z} w\right|^{2} \mathrm{~d} x \mathrm{~d} z-\int_{d}^{D}\left\{\partial_{x} w\left(r_{n}, z\right) \overline{w\left(r_{n}, z\right)}-\partial_{x} w\left(-r_{n}, z\right) \overline{w\left(-r_{n}, z\right)}\right\} \mathrm{d} z \\
& -\int_{-r_{n}}^{r_{n}}\left\{\partial_{z} w(x, D) \overline{w(x, D)}-\partial_{z} w(x, d) \overline{w(x, d)}\right\} \mathrm{d} x .
\end{aligned}
$$

Hence,

$$
\begin{aligned}
& \operatorname{Im} \int_{-r_{n}}^{r_{n}}\left\{\partial_{z} w(x, D) \overline{w(x, D)} \mathrm{d} x\right. \\
& \quad=\operatorname{Im} \int_{-r_{n}}^{r_{n}}\left\{\partial_{z} w(x, d) \overline{w(x, d)} \mathrm{d} x-\operatorname{Im} \int_{d}^{D}\left\{\partial_{x} w\left(r_{n}, z\right) \overline{w\left(r_{n}, z\right)}-\partial_{x} w\left(-r_{n}, z\right) \overline{w\left(-r_{n}, z\right)}\right\} \mathrm{d} z .\right.
\end{aligned}
$$

Letting $n \rightarrow \infty$, (5.10) implies that

$$
\int_{-\infty}^{\infty} \operatorname{Im}\left\{\partial_{z} w(x, D) \overline{w(x, D)} \mathrm{d} x=\int_{-\infty}^{\infty} \operatorname{Im}\left\{\partial_{z} w(x, d) \overline{w(x, d)}\right\} \mathrm{d} x, \quad \text { for all } D>d .\right.
$$




\subsection{Guided TE travelling waves}

For TE modes that are travelling waves, there exist $k>0$ and $W \in C^{2}(\mathbb{R}, \mathbb{C})$ such that $w(x, z)=$ $W(x) \mathrm{e}^{\mathrm{i} k z}$. In this case, (5.5) simplifies to

$$
W^{\prime \prime}(x)-k^{2} W(x)+\left(\frac{\omega}{c}\right)^{2} \mu\left(\omega, x, \frac{1}{2}|W(x)|^{2}\right) W(x)=0, \quad \text { for } x \in \mathbb{R},
$$

and the guidance conditions reduce to

$$
\text { (i) } W \in H^{1}(\mathbb{R}, \mathbb{C}) \text { and (ii) } W(x) \text { and } W^{\prime}(x) \rightarrow 0 \text { as }|x| \rightarrow \infty \text {. }
$$

The corresponding electromagnetic fields are

$$
E=\operatorname{Re}\left\{\left(\begin{array}{c}
0 \\
W(x) \mathrm{e}^{\mathrm{i}(k z-\omega t)} \\
0
\end{array}\right)\right\} \quad \text { and } \quad B=-\frac{c}{\omega} \operatorname{Re}\left\{\left(\begin{array}{c}
k W(x) \\
0 \\
\mathrm{i} W^{\prime}(x)
\end{array}\right) \mathrm{e}^{\mathrm{i}(k z-\omega t)}\right\} .
$$

The power of a TE travelling wave is

$$
P=\frac{c^{2}}{2 \omega} \int_{-\infty}^{\infty} \operatorname{Im}\left\{\overline{w(x, z)} \partial_{z} w(x, z)\right\} \mathrm{d} x=\frac{c^{2} k}{2 \omega} \int_{-\infty}^{\infty}|W(x)|^{2} \mathrm{~d} x .
$$

Setting $u(x)=\operatorname{Re} W(x)$ and $v(x)=\operatorname{Im} W(x)$, the complex equation (5.11) is equivalent to the real system

$$
\begin{aligned}
& u^{\prime \prime}-k^{2} u+\left(\frac{\omega}{c}\right)^{2} \mu\left(\omega, x, \frac{1}{2}\left[u(x)^{2}+v(x)^{2}\right]\right) u=0, \\
& v^{\prime \prime}-k^{2} v+\left(\frac{\omega}{c}\right)^{2} \mu\left(\omega, x, \frac{1}{2}\left[u(x)^{2}+v(x)^{2}\right]\right) v=0,
\end{aligned}
$$

but, in fact, as the next result shows, solutions of this system have a simple form that means it is enough to study the scalar equation

$$
U^{\prime \prime}-k^{2} U+\left(\frac{\omega}{c}\right)^{2} \mu\left(\omega, x, \frac{1}{2} U(x)^{2}\right) U(x)=0,
$$

for $U \in C^{2}(\mathbb{R}, \mathbb{R})$.

Proposition 5.2 Suppose that $\mu$ satisfies (3.4)-(3.6). A function $W \in C^{2}(\mathbb{R}, \mathbb{C})$ and satisfies (5.11) and (5.12) if and only if

$$
W(x)=\mathrm{e}^{\mathrm{i} \theta} U(x), \quad \text { for all } x \in \mathbb{R},
$$

for some $\theta \in[0,2 \pi)$ and $U \in H^{1}(\mathbb{R}, \mathbb{R})$ such that

$$
\begin{aligned}
& \int_{-\infty}^{\infty} U^{\prime}(x) \varphi^{\prime}(x) \mathrm{d} x \\
& \quad=\int_{-\infty}^{\infty}\left\{\left(\frac{\omega}{c}\right)^{2} \mu\left(\omega, x, \frac{1}{2} U(x)^{2}\right)-k^{2}\right\} U(x) \varphi(x) \mathrm{d} x, \quad \text { for all } \varphi \in C_{0}^{\infty}(\mathbb{R}, \mathbb{R}) .
\end{aligned}
$$


REMARK 5.1 Thus, $U$ is only required to be a weak solution of (5.17), but this implies that $U \in C^{2}$ $(\mathbb{R}, \mathbb{R})$ and satisfies $(5.17)$ in the classical sense. The function $U$ may change sign, but as a solution of (5.17), all its zeros are simple, except for the case $U \equiv 0$. The condition (5.18) means that the graph of $W$ lies on a plane in $\mathbb{R} \times \mathbb{R}^{2}$ when $\mathbb{C}$ is identified with $\mathbb{R}^{2}$.

REMARK 5.2 For $U \in H^{1}(\mathbb{R}, \mathbb{R})$, we have that (5.19) is satisfied for all $\varphi \in C_{0}^{\infty}(\mathbb{R}, \mathbb{R})$ if and only if it is satisfied for all $\varphi \in H^{1}(\mathbb{R}, \mathbb{R})$.

Proof. Suppose first that $W=u+\mathrm{i} v \in C^{2}(\mathbb{R}, \mathbb{C})$ and satisfies (5.11) and (5.12). It follows from (5.15) and (5.16) that $\partial_{x}\left\{u \partial_{x} v-v \partial_{x} u\right\} \equiv 0$ and hence that $u \partial_{x} v-v \partial_{x} u=C$ on $\mathbb{R}$ for some constant $C$. But (5.12) implies that $C=0$. If $v \equiv 0$, the result is trivial $(\theta=0$ and $U=u)$ so we suppose henceforth that $v \not \equiv 0$. It follows from (5.16) that the zeros of $v$ are isolated and that they are all simple. Let $(a, b)$ be a maximal interval on which $v \neq 0$. Then, $\partial_{x}\left\{\frac{u}{v}\right\} \equiv 0$ on $(a, b)$ and so there exists a constant $d$ such that $u \equiv d v$ on $(a, b)$. Setting $U(x)=\sqrt{1+d^{2} v(x)}$, (5.16) shows that $U$ satisfies (5.17). Choosing $\theta \in[0,2 \pi)$ such that $\mathrm{e}^{\mathrm{i} \theta}=(d+i) / \sqrt{d^{2}+1}$, we have that $W(x)=\mathrm{e}^{\mathrm{i} \theta} U(x)$ for all $x \in(a, b)$.

If $b<\infty$, let $(b, \beta)$ denote the next interval on which $v \neq 0$. Then as above, we have that $u \equiv d_{1} v$ on $(b, \beta)$ for some constant $d_{1}$. But this implies that

$$
\begin{aligned}
u^{\prime}(b-) & =d v^{\prime}(b-) \text { and } u^{\prime}(b+)=d_{1} v^{\prime}(b+), \\
\text { where } u^{\prime}(b-) & =u^{\prime}(b+) \text { and } v^{\prime}(b-)=v^{\prime}(b+) \neq 0,
\end{aligned}
$$

since $u, v \in C^{1}(\mathbb{R})$ and $b$ is a simple zero of $v$. This shows that $d_{1}=d$ and it follows by induction that $u(x)=d v(x)$ on $\mathbb{R}$.

Conversely, if $U \in H^{1}(\mathbb{R})$, it follows from (3.5) that $\left\{\left(\frac{\omega}{c}\right)^{2} \mu\left(\omega, x, \frac{1}{2} U(x)^{2}\right)-k^{2}\right\}$ is a bounded continuous function of $x$ on $\mathbb{R}$. From this and (5.19) we deduce that $U \in C^{2}(\mathbb{R}) \cap H^{2}(\mathbb{R})$ and satisfies (5.17). One easily checks that, for any $\theta, W(x)=\mathrm{e}^{\mathrm{i} \theta} U(x)$ has the required properties.

By Proposition 5.2, the study of all guided planar TE travelling waves has been reduced to finding $U \in H^{1}(\mathbb{R}, \mathbb{R})$ such that (5.17) holds in the weak sense. We now turn the results concerning the existence and stability of such travelling waves.

\section{Stability in the paraxial approximation}

In Section 5, we found that, for all planar TE travelling waves, the electric field can be expressed in the form

$$
E(x, y, z, t)=\operatorname{Re}\left\{\mathrm{e}^{\mathrm{i} \theta}(x) U(x) \mathrm{e}^{\mathrm{i}(k z-\omega t)}\right\} e_{2}=U(x) \cos (k z-\omega t+\theta) e_{2},
$$

where $U \in C^{2}(\mathbb{R}, \mathbb{R})$ satisfies (5.17) and $\theta \in[0,2 \pi)$. On the plane $z=0$, which is regarded as the extremity of a slab wave guide occupying the half-space $z>0$, the electric field is

$$
E(x, y, 0, t)=U(x) \cos (-\omega t+\theta) e_{2} .
$$

In considering the stability of this travelling wave, it is natural to inquire what happens if a device at the extremity $z=0$ stimulates an electric field $\widetilde{E}(x, y, 0, t)$ that is close to (6.2). Will the resulting electromagnetic fields that are generated in the wave guide remain close to the travelling wave for all $z>0$ and all $t$ ?

So far, this issue seems only to have been investigated in the context of the paraxial approximation, in which Maxwell's equations are simplified to some form of NLS. In this section, we give a derivation 
of this approximate equation in the context of planar TE modes and then formulate the appropriate notion of stability.

For any guided TE mode, the electric field can be written as

$$
E(x, y, z, t)=\operatorname{Re}\left\{w(x, z) \mathrm{e}^{-\mathrm{i} \omega t}\right\} e_{2},
$$

where $w \in C^{2}\left(\mathbb{R}^{2}, \mathbb{C}\right)$ satisfies the elliptic equation (5.5) and the guidance conditions (5.7) and (5.8). Let $\lambda_{0}$ be some typical (and hence, $\lambda_{0} \ll 1$ ) value of the wavelength of light and let $k_{0}=2 \pi / \lambda_{0}$ denote the corresponding wave number, $k_{0} \gg 1$. Without any loss of generality, the function $w(x, z)$ can be written as

$$
w(x, z)=W(x, z) \mathrm{e}^{\mathrm{i} k_{0} z}
$$

and (5.5) becomes

$$
\Delta W(x, z)+2 \mathrm{i} k_{0} \partial_{z} W(x, z)-k_{0}^{2} W(x, z)+\left(\frac{\omega}{c}\right)^{2} \mu\left(\omega, x, \frac{1}{2}|W(x, z)|^{2}\right) W(x, z)=0, \quad \text { for }(x, z) \in \mathbb{R}^{2},
$$

whereas $W$ still satisfies same conditions (5.7) and (5.8). In this notation, the power (5.9) becomes

$$
\begin{aligned}
P & =\frac{c^{2}}{2 \omega} \int_{-\infty}^{\infty} \operatorname{Im}\left\{\overline{W(x, z)}\left[\partial_{z} W(x, z)+i k_{0} W(x, z)\right]\right\} \mathrm{d} x \\
& =\frac{c^{2}}{2 \omega} \int_{-\infty}^{\infty} \operatorname{Im}\left\{\overline{W(x, z)} \partial_{z} W(x, z)\right\} \mathrm{d} x+\frac{c^{2} k_{0}}{2 \omega} \int_{-\infty}^{\infty}|W(x, z)|^{2} \mathrm{~d} x .
\end{aligned}
$$

In the 'paraxial approximation' (see Saleh \& Teich, 1991, p. 50, e.g.) it is supposed that $W(x, z)$ is a 'slowly varying function' of $z$ in the sense that $\partial_{z} W(x, z)$ and $\partial_{z z}^{2} W(x, z)$ can be neglected when compared to $k_{0} W(x, z)$ and $k_{0}^{2} W(x, z)$, respectively. In this approximation, the exact equation (6.4) is replaced by

$$
\partial_{x x}^{2} W(x, z)+2 \mathrm{i} k_{0} \partial_{z} W(x, z)-k_{0}^{2} W(x, z)+\left(\frac{\omega}{c}\right)^{2} \mu\left(\omega, x, \frac{1}{2}|W(x, z)|^{2}\right) W(x, z)=0
$$

and the power by

$$
P(z)=\frac{c^{2} k_{0}}{2 \omega} \int_{-\infty}^{\infty}|W(x, z)|^{2} \mathrm{~d} x .
$$

As we shall see later in Proposition 7.2, the quantity (6.7) is independent of $z$ whenever $W$ satisfies (6.6) and the guidance conditions (5.7) and (5.8). Furthermore, prescribing the electric field at the extremity $z=0$ of the wave guide is equivalent to prescribing $W(x, 0)$. Equation (6.6) has the form of a NLS and, under appropriate assumptions on the response function $\mu(\omega, \cdot)$, the corresponding initial-value problem is well-posed so one can meaningfully investigate the stability of a solution determined by a particular choice of initial condition. We consider the initial conditions that generate guided TE travelling waves, namely

$$
W(x, 0)=w(x, 0)=\mathrm{e}^{\mathrm{i} \theta} U(x),
$$

where $\theta \in[0,2 \pi)$ and $U \in C^{2}(\mathbb{R}, \mathbb{R}) \cap H^{1}(\mathbb{R}, \mathbb{R})$ satisfies (5.17) for some $k>0$. In this case, the exact solution of (6.4) is

$$
w(x, z)=\mathrm{e}^{\mathrm{i} \theta} U(x) \mathrm{e}^{\mathrm{i} k z}
$$


and the electric field is

$$
E(x, y, z, t)=U(x) \cos (k z-\omega t+\theta) e_{2} .
$$

Substitution shows that the solution of (6.6) defined by the initial condition (6.8) is the following $z$-periodic function

$$
W(x, z)=\mathrm{e}^{\mathrm{i} \theta} U(x) \mathrm{e}^{\mathrm{i} \lambda_{k} z}, \quad \text { where } \lambda_{k}=\frac{k^{2}-k_{0}^{2}}{2 k_{0}} .
$$

Observe that $W$ is a slowly varying function of $z$ provided that

$$
\left|\frac{k^{2}-k_{0}^{2}}{2 k_{0}}\right| \ll k_{0}
$$

The corresponding electric field is

$$
E(x, y, z, t)=\operatorname{Re}\left\{W(x, z) \mathrm{e}^{\mathrm{i}\left(k_{0} z-\omega t\right)}\right\} e_{2}=U(x) \cos \left(\left[\lambda_{k}+k_{0}\right] z-\omega t+\theta\right) e_{2} .
$$

The difference between the wave number $\lambda_{k}+k_{0}$ of this field and the wave number $k$ in the exact expression (6.9) is

$$
\lambda_{k}+k_{0}-k=\frac{\left(k-k_{0}\right)^{2}}{2 k_{0}}
$$

and this quantity is negligible for $k$ near $k_{0}$ and $k_{0}>>1$.

\subsection{Stability}

Thus, we are lead to investigate the stability of the solution (6.10) of the NLS (6.6) with respect to perturbations of its initial condition (6.8). By Proposition 7.2 below, $H^{1}(\mathbb{R}, \mathbb{C})$ is the appropriate phase space for this initial-value problem and we denote by $\|\cdot\|_{H^{1}}$ the usual norm

$$
\|W(\cdot, z)\|_{H^{1}}=\left[\int_{-\infty}^{\infty}|W(x, z)|^{2}+\left|\partial_{x} W(x, z)\right|^{2} \mathrm{~d} x\right]^{1 / 2}
$$

of $W(\cdot, z)$ in this space. Note that this choice of phase space is also well-adapted to the guidance condition (5.7).

Let $\theta_{n} \rightarrow \theta$ and let

$$
W_{n}(x, z)=\mathrm{e}^{\mathrm{i} \theta_{n}} U(x) \mathrm{e}^{\mathrm{i} \lambda_{k} z} \quad \text { and } \quad W(x, z)=\mathrm{e}^{\mathrm{i} \theta} U(x) \mathrm{e}^{\mathrm{i} \lambda_{k} z} .
$$

Clearly, $\left\|W_{n}(\cdot, 0)-W(\cdot, 0)\right\|_{H^{1}}=\left|\mathrm{e}^{\mathrm{i} \theta_{n}}-\mathrm{e}^{\mathrm{i} \theta}\right|\|U\|_{H^{1}} \rightarrow 0$ as $n \rightarrow \infty$, but

$$
\left\|W_{n}(\cdot, z)-W(\cdot, z)\right\|_{H^{1}}=\left|\mathrm{e}^{\mathrm{i} \theta_{n}}-\mathrm{e}^{\mathrm{i} \theta}\right|\|U\|_{H^{1}} \nrightarrow \rightarrow 0 \quad \text { as } z \rightarrow \infty,
$$

for any $n$ and so we cannot expect to establish asymptotic stability of $W$. Furthermore, if we consider a sequence $\left\{\left(k_{n}, U_{n}\right)\right\}$ of solutions of (5.17) and (5.7) such that $k_{n} \rightarrow k$ and $\left\|U_{n}-U\right\|_{H^{1}} \rightarrow 0$ (the existence of such a sequence is established in Theorem 7.1), and now set

$$
W_{n}(x, z)=\mathrm{e}^{\mathrm{i} \theta} U_{n}(x) \mathrm{e}^{\mathrm{i} \lambda_{k_{n}} z},
$$


then again $\left\|W_{n}(\cdot, 0)-W(\cdot, 0)\right\|_{H^{1}}=\left\|U_{n}-U\right\|_{H^{1}} \rightarrow 0$. But

$$
W_{n}(x, z)-W(x, z)=\mathrm{e}^{\mathrm{i} \theta}\left[U_{n}(x)-U(x)\right] \mathrm{e}^{\mathrm{i} \lambda_{k_{n}} z}+\mathrm{e}^{\mathrm{i} \theta} U(x)\left[\mathrm{e}^{\mathrm{i} \lambda_{k_{n}} z}-\mathrm{e}^{\mathrm{i} \lambda_{k} z}\right],
$$

and so

$$
\left|W_{n}(x, z)-W(x, z)\right| \geqslant|U(x)|\left|\mathrm{e}^{\mathrm{i}\left[\lambda_{k_{n}}-\lambda_{k}\right] z}-1\right|-\left|U_{n}(x)-U(x)\right|
$$

showing that

$$
\lim \sup _{z \geqslant 0}\left|W_{n}(x, z)-W(x, z)\right| \geqslant 2|U(x)|-\left|U_{n}(x)-U(x)\right|,
$$

if $k_{n} \neq k$. Thus, even stability fails in this case. For these reasons, we concentrate on the orbital stability of $W$ as a solution of (6.6).

\subsection{Orbital stability of guided TE travelling waves}

The orbit $\Theta\left(\mathrm{e}^{\mathrm{i} \theta} U(\cdot)\right)$ of the solution $(6.10)$ in the phase space $H^{1}(\mathbb{R}, \mathbb{C})$ is

$$
\Theta\left(\mathrm{e}^{\mathrm{i} \theta} U(\cdot)\right)=\left\{\mathrm{e}^{\mathrm{i} \theta} U(\cdot) \mathrm{e}^{\mathrm{i} \lambda_{k} z}: z \geqslant 0\right\}=\left\{\mathrm{e}^{\mathrm{i} \theta} U(\cdot): \theta \in \mathbb{R}\right\}=\Theta(U) .
$$

For any $V \in H^{1}(\mathbb{R}, \mathbb{C})$, let

$$
d\left(V, \Theta(U(\cdot))=\inf \left\{\|V-W\|_{H^{1}}: W \in \Theta(U(\cdot))\right\} .\right.
$$

Since $\Theta(U(\cdot))$ is a compact subset of $H^{1}(\mathbb{R}, \mathbb{C})$, there exists $W \in \Theta(U(\cdot))$ such that $d(V, \Theta(U(\cdot))=$ $\|V-W\|_{H^{1}}$, i.e. there exists $z \geqslant 0$ such that

$$
d\left(V, \Theta(U(\cdot))=\left\|V-\mathrm{e}^{\mathrm{i} \theta} U(\cdot) \mathrm{e}^{\mathrm{i} \lambda_{k} z}\right\|_{H^{1}} .\right.
$$

Let $W(\cdot, z)$ denote the solution of (6.6) having an arbitrary initial condition $W(\cdot, 0) \in H^{1}(\mathbb{R}, \mathbb{C})$. 'Orbital stability' of the $z$-periodic solution (6.10) means that, given any $\varepsilon>0$, there exists $\delta>0$ such that

$$
\left\|W(\cdot, 0)-\mathrm{e}^{\mathrm{i} \theta} U(\cdot)\right\|_{H^{1}}<\delta \Longrightarrow d\left(W(\cdot, z), \Theta\left(\mathrm{e}^{\mathrm{i} \theta} U(\cdot)\right)\right)<\varepsilon, \quad \text { for all } z \geqslant 0 .
$$

That is, for all $z \geqslant 0$,

$$
\text { there exists } z_{1} \geqslant 0 \text { such that }\left\|W(\cdot, z)-\mathrm{e}^{\mathrm{i} \theta} U(\cdot) \mathrm{e}^{\mathrm{i} \lambda_{k} z_{1}}\right\|_{H^{1}}<\varepsilon,
$$

or, equivalently,

$$
\text { there exists } \theta(z)\left(=\theta+\lambda_{k} z_{1}\right) \text { such that }\left\|W(\cdot, z)-\mathrm{e}^{\mathrm{i} \theta(z)} U(\cdot)\right\|_{H^{1}}<\varepsilon .
$$

Of course, a complete statement of this notion of stability should include the requirement that (6.6) has a unique global solution $W(\cdot, z)$ for all initial conditions $W(\cdot, 0)$ close to $\Theta\left(\mathrm{e}^{\mathrm{i} \theta} U(\cdot)\right)$. This will be done in Section 7 when we formulate results concerning orbital stability.

Observing that $\Theta\left(\mathrm{e}^{\mathrm{i} \theta} U(\cdot)\right)=\Theta(U(\cdot))$ for all $\theta$, the above definition is equivalent to requiring that, for all $\varepsilon>0$, there exists $\delta>0$ such that

$$
d(W(\cdot, 0), \Theta(U(\cdot)))<\delta \Longrightarrow d(W(\cdot, z), \Theta(U(\cdot))), \quad \text { for all } z \geqslant 0 .
$$


To bring out the physical content of this kind of stability, we consider the behaviour of the amplitude and the phase of $W(x, z)$. Let

$$
A(x, z)=|W(x, z)| \quad \text { and } \quad W(x, z)=A(x, z) \mathrm{e}^{\mathrm{i} \varphi(x, z)} .
$$

Recalling that $U(x) \in \mathbb{R}$, we have that $U(x)=|U(x)| \mathrm{e}^{\mathrm{i} \psi(x)}$, where $\psi(x)=0$ or $\pi$. In terms of the electric fields, we have that the field produced by the perturbed initial condition $E(x, y, 0, t)=$ $\operatorname{Re}\left\{W(x, 0) \mathrm{e}^{-\mathrm{i} \omega t}\right\} e_{2}$ is

$$
E(x, y, z, t)=\operatorname{Re}\left\{W(x, z) \mathrm{e}^{\mathrm{i}\left(k_{0} z-\omega t\right)}\right\} e_{2}=A(x, z) \cos \left(k_{0} z-\omega t+\varphi(x, z)\right) e_{2},
$$

whereas the unperturbed travelling wave is

$$
\widetilde{E}(x, y, z, t)=\operatorname{Re}\left\{\mathrm{e}^{\mathrm{i} \theta} U(x) \mathrm{e}^{\mathrm{i} \lambda_{k} z} \mathrm{e}^{\mathrm{i}\left(k_{0} z-\omega t\right)}\right\} e_{2}=|U(x)| \cos \left(k_{0} z-\omega t+\chi(x, z)\right) e_{2},
$$

where $\chi(x, z)=\theta+\lambda_{k} z+\psi(x)$. From (6.11) we have that, for all $z \geqslant 0$, there exists $z_{1} \geqslant 0$ such that $\left\|W(\cdot, z)-\mathrm{e}^{\mathrm{i} \theta} U(\cdot) \mathrm{e}^{\mathrm{i} \lambda_{k} z_{1}}\right\|_{H^{1}}<\varepsilon$, from which it follows that

$$
\sup _{x \in \mathbb{R}}\left|A(x, z) \mathrm{e}^{\mathrm{i} \varphi(x, z)}-\mathrm{e}^{\mathrm{i} \chi\left(x, z_{1}\right)}\right| U(x)||=\sup _{x \in \mathbb{R}}\left|A(x, z) \mathrm{e}^{\mathrm{i}\left[\varphi(x, z)-\chi\left(x, z_{1}\right)\right]}-\right| U(x)||<\varepsilon .
$$

Thus, orbital stability implies that that the amplitudes $A(x, z)$ and $|U(x)|$ are close for all $x$ and $z$. Furthermore, the phase $\varphi(x, z)$ of $W(x, z)$ is almost independent of $x$ on intervals where $U$ is bounded away from zero.

\section{Existence and stability of symmetric guided TE travelling waves}

Before continuing, let us summarize the problems that have been formulated. The dielectric response function $\mu$ is supposed to have the following properties.

(A) $\mu:(a, b) \times \mathbb{R} \times[0, \infty) \rightarrow \mathbb{R}$ where, for $\omega \in(a, b) \subset(0, \infty)$,

(i) $f \in C^{1}\left(\mathbb{R}^{2}\right)$, where $f(x, s)=\mu\left(\omega, x, \frac{1}{2} s^{2}\right) s$,

(ii) $\inf \{\mu(\omega, x, s): x \in \mathbb{R}$ and $s \geqslant 0\}>0$,

(iii) for any $K>0, \mu(\omega, \cdot, \cdot)$ is bounded and uniformly continuous on $\mathbb{R} \times[0, K]$.

In fact, in (3.4) we made the stronger assumption that $\mu(\omega, \cdot, \cdot) \in C^{1}(\mathbb{R} \times[0, \infty))$ but it is easily checked that (7.1) is sufficient when dealing with TE modes.

Existence: Find $k>0$ and $U \in H^{1}(\mathbb{R})$ such that (5.19) is satisfied.

Solutions of this problem furnish all the exact solutions of Maxwell's equations that are guided by the planar monochromatic TE travelling waves through the formulae

$$
\begin{aligned}
& E(x, y, z, t)=U(x) \cos (k z-\omega t+\theta) e_{2} \text { and } \\
& B(x, y, z, t)=-\frac{c k}{\omega} U(x) \cos (k z-\omega t+\theta) e_{1}+\frac{c}{\omega} U^{\prime}(x) \sin (k z-\omega t+\theta) e_{3},
\end{aligned}
$$

where $\theta \in[0,2 \pi)$ is arbitrary. Furthermore, for any solution $(k, U)$ of this problem, the function $W$ defined by (6.10) is an exact solution of the initial-value problem for NLS (6.6) with the initial condition (6.8). 
Stability: Discuss the orbital stability of the standing wave defined by (6.10) as a solution of (6.6) with respect to perturbations of its initial condition. In fact, we have seen that it is sufficient to deal with the case $\theta=0$.

\subsection{Canonical form of the equations}

In order to present some of the results concerning these problems, it is convenient to reduce the equations to a more canonical, but equivalent, form. First we set

$$
\theta=0, \quad \tau=\frac{z}{2 k_{0}} \quad \text { and then } \quad \Psi(x, \tau)=W(x, z)=W\left(x, 2 k_{0} \tau\right),
$$

so that (6.6) and (6.10) become, for $x \in \mathbb{R}$ and $\tau>0$

$$
\begin{aligned}
& \mathrm{i} \partial_{\tau} \Psi(x, \tau)+\partial_{x x}^{2} \Psi(x, \tau)-k_{0}^{2} \Psi(x, \tau)+\left(\frac{\omega}{c}\right)^{2} \mu\left(\omega, x, \frac{1}{2}|\Psi(x, \tau)|^{2}\right) \Psi(x, \tau)=0, \\
& \Psi(x, 0)=U(x), \quad \text { for } x \in \mathbb{R} .
\end{aligned}
$$

Next, we set

$$
\begin{aligned}
& L(\infty)=\lim \inf _{|x| \rightarrow \infty}\left(\frac{\omega}{c}\right)^{2} \mu(\omega, x, 0), \quad \gamma=L(\infty)-k_{0}^{2}, \quad \lambda=L(\infty)-k^{2}, \\
& V(x)=\left(\frac{\omega}{c}\right)^{2} \mu(\omega, x, 0)-L(\infty), \quad g(x, s)=\left(\frac{\omega}{c}\right)^{2}\left\{\mu\left(\omega, x, \frac{1}{2} s\right)-\mu(\omega, x, 0)\right\},
\end{aligned}
$$

so that (7.4) becomes

$$
\partial_{x x}^{2} \Psi(x, \tau)+\mathrm{i} \partial_{\tau} \Psi(x, \tau)+[V(x)+\gamma] \Psi(x, \tau)+g\left(x,|\Psi(x, \tau)|^{2}\right) \Psi(x, \tau)=0 .
$$

Then, we set $\Phi(x, \tau)=\Psi(x, \tau) \mathrm{e}^{-\mathrm{i} \gamma \tau}$ so that (7.8) becomes

$$
\mathrm{i} \partial_{\tau} \Phi(x, \tau)+\partial_{x x}^{2} \Phi(x, \tau)+V(x) \Phi(x, \tau)+g\left(x,|\Phi(x, \tau)|^{2}\right) \Phi(x, \tau)=0 .
$$

Finally in the new variables $\tau$ and $\Phi$, where

$$
\tau=\frac{z}{2 k_{0}} \quad \text { and } \quad \Phi(x, \tau)=W\left(x, 2 k_{0} \tau\right) \mathrm{e}^{-\mathrm{i} \gamma \tau}=W(x, z) \mathrm{e}^{-\mathrm{i} \frac{\gamma}{2 k_{0}} z},
$$

the standing wave $U(x) \mathrm{e}^{\mathrm{i} \lambda_{k} z}$ generated by $(k, U)$ becomes

$$
\Phi(x, \tau)=U(x) \mathrm{e}^{\mathrm{i} \lambda_{k} z} \mathrm{e}^{-\mathrm{i} \frac{\gamma}{2 k_{0}} z}=U(x) \mathrm{e}^{\mathrm{i}\left[k^{2}-L(\infty)\right] \tau}=U(x) \mathrm{e}^{-\mathrm{i} \lambda \tau},
$$

since $\lambda_{k}=\frac{k^{2}-k_{0}^{2}}{2 k_{0}}$ and $\gamma=L(\infty)-k_{0}^{2}$. Furthermore, in this notation, (5.19) can be written as

$$
\int_{-\infty}^{\infty} U^{\prime}(x) \varphi^{\prime}(x) \mathrm{d} x=\int_{-\infty}^{\infty}\left\{V(x)+g\left(x, U(x)^{2}\right)+\lambda\right\} U(x) \varphi(x) \mathrm{d} x, \quad \text { for all } \varphi \in C_{0}^{\infty}(\mathbb{R}, \mathbb{R}) .
$$

Using the notation (7.7), we can express the problems of existence and stability in the following equivalent way. 
Suppose that the functions $V: \mathbb{R} \rightarrow(0, \infty)$ and $g: \mathbb{R} \times[0, \infty) \rightarrow \mathbb{R}$ have the following properties:

(H1) $V \in C^{1}(\mathbb{R}) \cap L^{\infty}(\mathbb{R})$ and $\liminf _{|x| \rightarrow \infty} V(x)=0$.

(H2) For all $K>0, g$ is bounded and uniformly continuous on $\mathbb{R} \times[0, K]$.

(H3) $g(x, 0)=0$ for all $x \in \mathbb{R}$ and $\inf \{g(x, s): x \in \mathbb{R}$ and $s \geqslant 0\}>-\infty$.

(H4) $f \in C^{1}\left(\mathbb{R}^{2}\right)$ where $f(x, s)=g\left(x, s^{2}\right) s$.

For a dielectric response $\mu$ that satisfies (A), the functions $V$ and $g$ defined by (7.7) have these properties. Conversely, given two functions $V$ and $g$ that satisfy (H1)-(H4), there exists a constant $L_{0}>0$ such that the function defined by

$$
\mu(\omega, x, s)=\left(\frac{c}{\omega}\right)^{2}\{L+V(x)+g(x, 2 s)\}
$$

satisfies the condition (A) for all $L>L_{0}$. Furthermore, $L(\infty)=L$.

Given $V$ and $g$ satisfying (H1)-(H4), the basic problems now take the following form.

Existence: Find $\lambda<L(\infty)$ and $U \in H^{1}(\mathbb{R})$ such that (7.12) is satisfied.

For any solution $(\lambda, U)$ of this problem, the function $\Phi(x, \tau)=U(x) \mathrm{e}^{-\mathrm{i} \lambda \tau}$ is an exact solution of the initial-value problem for the NLS (7.9) with $\Phi(x, 0)=U(x)$.

Stability: Discuss the orbital stability of the $\tau$-periodic solution $U(x) \mathrm{e}^{-\mathrm{i} \lambda \tau}$ as a solution of (7.9) with respect to perturbations of its initial condition.

REMARK 7.1 The assumptions (H1)-(H4), and consequently the condition (A) in the case of a homogeneous self-focusing medium, are satisfied by

$$
V \equiv 0 \text { and } g(x, s)=G(s) \text {, where } G \in G^{1}([0, \infty)) \text { with } G(0)=0 \text { and } G^{\prime}(s)>0 \text {, for } s>0 .
$$

As is shown in Remark 8.3.3 of Cazenave (2003), a standing wave $U(x) \mathrm{e}^{-\mathrm{i} \lambda \tau}$ cannot be orbital stability in this case due to the fact that the problem is then invariant with respect to translations in $x$. A weaker notion of stability is introduced to deal with this situation (Cazenave \& Lions, 1982).

\subsection{A branch of TE travelling waves in a self-focusing wave guide}

To obtain a smooth branch of TE travelling waves we make the following hypotheses.

(E1) $V \in C^{1}(\mathbb{R})$ is an even function with

$$
V^{\prime}(x) \leqslant 0, \text { for all } x \geqslant 0, \quad \text { and } \quad V(0)>0=\lim _{x \rightarrow \infty} V(x)
$$

(E2) $g \in C(\mathbb{R} \times[0, \infty)) \cap C^{1}(\mathbb{R} \times(0, \infty))$ with $g(x, 0)=0$ and $F \in C^{1}\left(\mathbb{R}^{2}\right)$ where $F(x, s)=$ $g\left(x, s^{2}\right) s$. Furthermore, for all $K>0, g$ and $\partial_{2} F$ are bounded and uniformly continuous on $\mathbb{R} \times[0, K]$.

(E3) For all $x \geqslant 0$ and $s>0$,

$$
g(x, s)=g(-x, s), \quad \partial_{1} g(x, s) \leqslant 0 \quad \text { and } \quad \partial_{2} g(x, s)>0 .
$$

It follows from these assumptions that (H1)-(H4) are satisfied with $V(x) \geqslant 0$ and $g(x, s)>0$ for all $x \in \mathbb{R}$ and $s>0$. Thus, the corresponding response function defined by (7.13) satisfies the condition (A) for any $L>0$. By (E3), the response is everywhere self-focusing. 
Set

$$
g(\infty, s)=\lim _{x \rightarrow \infty} g(x, s), \quad \text { for } s \geqslant 0 .
$$

Clearly, $0 \leqslant g(\infty, s) \leqslant g(x, s) \leqslant g(0, s)$ for all $x \in \mathbb{R}$ and $s \geqslant 0$. Also $g(\infty, s)$ is a non-decreasing function of $s$. It will be useful to distinguish the following two cases.

(L1) $\lim _{s \rightarrow \infty} g(\infty, s)=\infty$.

(L2) There exists $P \in L^{\infty}(0, \infty)$ such that $\lim _{s \rightarrow \infty} g(x, s)=P(x)$, uniformly for $x$ in compact intervals.

EXAMPLE 1 For $A>0, \sigma>0$ and $\alpha \geqslant 0$, the function

$$
g(x, s)=A\left(1+x^{2}\right)^{-\alpha} s^{\sigma}
$$

satisfies the conditions (E2) and (E3). For $\alpha=0$, the case (L1) occurs. Note that for $0<\sigma<1, g(x, \cdot)$ is not differentiable at $s=0$, but $f(x, \cdot)$ is.

EXAMPLE 2 The function

$$
g(x, s)=\frac{s}{1+s}
$$

also satisfies the conditions (E2) and (E3). Here, the case (L2) occurs with $P \equiv 1$.

By (E1), we can define a self-adjoint operator $S: H^{2}(\mathbb{R}) \subset L^{2}(\mathbb{R}) \rightarrow L^{2}(\mathbb{R})$ by

$$
S u=-u^{\prime \prime}-V u, \quad \text { for } u \in H^{2}(\mathbb{R}) .
$$

Denoting its spectrum and essential spectrum by $\sigma(S)$ and $\sigma_{e}(S)$, respectively, we have that

$$
-\infty<\Lambda=\inf \sigma(S)<0 \quad \text { and } \quad \sigma_{e}(S)=[0, \infty)
$$

Furthermore $\Lambda$ is a simple eigenfunction of $S$ with an eigenfunction $\varphi \in H^{2}(\mathbb{R}) \cap C^{3}(\mathbb{R})$ that has the following properties

$$
\varphi(x)=\varphi(-x)>0, \text { for all } x \in \mathbb{R}, \quad \text { and } \quad \varphi^{\prime}(x)<0, \text { for all } x>0 .
$$

This implies that $\Lambda$ is a bifurcation point for non-trivial solutions of (7.12), but one can say much more.

THEOREM 7.1 Let the conditions (E1)-(E3) be satisfied. Then

(i) there exist $\lambda^{*}<\Lambda$ and $u \in C^{1}\left(\left(\lambda^{*}, \Lambda\right), H^{2}(\mathbb{R})\right)$ such that $(\lambda, u(\lambda))$ satisfies (7.12) for all $\lambda \in\left(\lambda^{*}, \Lambda\right)$

(ii) for each $\lambda \in\left(\lambda^{*}, \Lambda\right)$ and with $u_{\lambda}=u(\lambda)$;

$$
\begin{aligned}
u_{\lambda}(x) & =u_{\lambda}(-x)>0, \text { for all } x \in \mathbb{R}, \quad \text { and } u_{\lambda}^{\prime}(x)<0, \text { for all } x>0, \\
\frac{\mathrm{d}}{\mathrm{d} \lambda} u_{\lambda}(0) & =\frac{\mathrm{d}}{\mathrm{d} \lambda}\left\|u_{\lambda}\right\|_{L^{\infty}}<0,
\end{aligned}
$$

(iii) we also have that

$$
\lim _{\lambda \rightarrow \Lambda}\left\|u_{\lambda}\right\|_{H^{2}}=0 \quad \text { and } \quad \lim _{\lambda \rightarrow \lambda^{*}}\left\|u_{\lambda}\right\|_{H^{2}}=\infty
$$


If, in addition,

(E4) there exist $\sigma>0, \gamma>0$ and $A \in C^{1}(\mathbb{R})$ such that

$$
\lim _{s \rightarrow 0} \frac{g(x, s)}{s^{\sigma}}=A(x) \geqslant \gamma, \quad \text { for } x \in \mathbb{R}
$$

then

$$
\lim _{\lambda \rightarrow \lambda^{*}}\left\|u_{\lambda}\right\|_{L^{\infty}}=\infty \text { where } \lambda^{*}=-\infty \text { if (L1) holds and } \lambda^{*}>-\infty \text { if (L2) holds. }
$$

Furthermore, if $(\lambda, U)$ satisfies (7.12) with $U(x) \geqslant 0$ for all $x$, but $U \not \equiv 0$, then $\lambda<\Lambda$ and $U=u_{\lambda}$.

Proof. See Jeanjean \& Stuart (1999).

REMARK 7.2 It is only the requirement $V(0)>\lim _{x \rightarrow \infty} V(x)$ in assumption (E1) that excludes the case of a homogeneous medium and thus ensures that positive solutions $U$ of (7.12) must have their maximum value at $x=0$. Note that (E2)-(E4) can be satisfied by a function $g$ of the type (7.14). Theorem 7.1 gives a complete description of all positive solutions of (7.12) and, as is shown in Stuart (2006), these solutions have a variational characterization as 'ground states'. For the homogeneous case (7.14), it is easy to show that solutions of (7.12) cannot change sign. On the other hand, under the hypotheses (E1)-(E4), the existence of sign-changing solutions is established in Ruppen (1997). The hypotheses also ensure that all the positive solution $u_{\lambda}$ have the same symmetry as the eigenfunction $\varphi$ of $S$. As is shown in Arcoya et al. (1999), in a symmetric waveguide, that is for functions $V$ and $g$ that are even with respect to $x,(7.12)$ may have positive asymmetric ground states.

\subsection{Orbital stability of the TE travelling waves}

To ensure global existence of solutions of the initial-value problem for (7.9), we introduce the following condition on $g$.

(S1) There exist constants $C>0$ and $\alpha \in[0,2)$ such that

$$
|g(x, s)| \leqslant C\left(1+s^{\alpha}\right), \quad \text { for all } x \in \mathbb{R} \text { and } s \geqslant 0 .
$$

The function defined by (7.15) satisfies (S1) if and only if $\sigma<2$. The function defined by (7.16) satisfies (S1) with $\alpha=0$.

Proposition 7.2 Let the conditions (E1), (E2) and (S1) be satisfied. Then, for any initial condition $\Phi_{0} \in H^{1}(\mathbb{R}, \mathbb{C})$, there exists a unique function $\Phi \in C\left([0, \infty), H^{1}(\mathbb{R}, \mathbb{C})\right) \cap C^{1}\left([0, \infty), H^{-1}(\mathbb{R}, \mathbb{C})\right)$ such that $\Phi$ satisfies (7.10) and $\Phi(\cdot, 0)=\Phi_{0}$. Furthermore,

$$
\int_{-\infty}^{\infty}|\Phi(x, \tau)|^{2} \mathrm{~d} x=\int_{-\infty}^{\infty}\left|\Phi_{0}(x)\right|^{2} \mathrm{~d} x, \quad \text { for all } \tau \geqslant 0 .
$$

Proof. See Cazenave (2003), Section 3.5.

REMARK 7.3 We note in passing that in the paraxial approximation, the expression (6.5) for the power of the electromagnetic fields becomes (6.7):

$$
P(z)=\frac{c^{2} k_{0}}{2 \omega} \int_{-\infty}^{\infty}|W(x, z)|^{2} \mathrm{~d} x=\frac{c^{2} k_{0}}{2 \omega} \int_{-\infty}^{\infty}|\Phi(x, \tau)|^{2} \mathrm{~d} x, \quad \text { where } \tau=\frac{z}{2 k_{0}},
$$

so (7.17) plays the role of Proposition 5.1 in the paraxial approximation. 
In order to ensure the orbital stability of all the standing waves generated by the whole branch of solutions $\left(\lambda, u_{\lambda}\right)$ given by Theorem 7.1, we need some extra assumptions about $g$.

(S2) For fixed $s>0, \partial_{2} g(x, s)$ is a non-increasing function of $x$ on $[0, \infty)$ and, for fixed $x \in \mathbb{R}$, $s \partial_{2} g(x, s)$ is a non-decreasing function of $s$ on $[0, \infty)$.

Set

$$
Q(x, s)=\frac{2 g(x, s)+x \partial_{1} g(x, s)}{s \partial_{2} g(x, s)}-1 .
$$

(S3) For fixed $s>0, Q(x, s)$ is a non-negative, non-increasing function of $x$ on $[0, \infty)$ and, for fixed $x \in \mathbb{R}, Q(x, s)$ is a non-decreasing function of $s$ on $[0, \infty)$.

By $(\mathrm{S} 2), s \partial_{2} g(x, s) \geqslant \partial_{2} g(x, 1)>0$ for all $s \geqslant 1$ and so $g(x, s) \geqslant \partial_{2} g(x, 1) \ln s$ for all $s \geqslant 1$. Hence, $\lim _{s \rightarrow \infty} g(x, s)=\infty$ for all $x \in \mathbb{R}$, and so (L2) cannot occur. Thus, (7.16) does not satisfy (S2).

The function defined by (7.15) satisfies (S2). Furthermore, in this case

$$
Q(x, s)=\frac{1}{\sigma}\left\{2-\sigma-2 \alpha+\frac{2 \alpha}{1+x^{2}}\right\},
$$

and so (S3) is satisfied if and only if $0<\sigma \leqslant 2$ and $0 \leqslant \alpha \leqslant 1-\frac{\sigma}{2}$.

THEOREM 7.3 Let the conditions (E1)- (E3) and (S1)-(S3) be satisfied and let $\left\{\left(\lambda, u_{\lambda}\right): \lambda \in\left(\lambda^{*}, \Lambda\right)\right\}$ be the branch of solutions of (7.12) given by Theorem 7.1. Then, for all $\lambda \in\left(\lambda^{*}, \Lambda\right)$, the standing wave $u_{\lambda}(x) \mathrm{e}^{-\mathrm{i} \lambda \tau}$ is an orbitally stable solution of (7.9) in the sense that, for all $\varepsilon>0$, there exists $\delta>0$ such that, for all initial conditions $\Phi_{0} \in H^{1}(\mathbb{R}, \mathbb{C})$ with $\left\|\Phi_{0}-u_{\lambda}\right\|_{H^{1}}<\delta$, the unique solution $\Phi$ of (7.9) given by Proposition 7.2 has the property that, for all $\tau \geqslant 0$, there exists $\theta(\tau) \in[0,2 \pi)$ such that

$$
\left\|\Phi_{0}(\cdot, \tau)-\mathrm{e}^{\mathrm{i} \theta(\tau)} u_{\lambda}(\cdot)\right\|_{H^{1}}
$$

Proof. See McLeod et al. (2003) and Stuart (2006).

EXAMPLE 3 The function defined by (7.15) satisfies all the conditions of this theorem if $0<\sigma<2$ and $0 \leqslant \alpha \leqslant 1-\frac{\sigma}{2}$. This includes the case of a Kerr medium since $\sigma=1$ in that case.

REMARK 7.4 There are many results establishing the orbital stability of standing wave solutions of more general NLSs in higher space dimensions. However, these results are either of a perturbative nature (Grillakis et al., 1987; Strauss, 1989; Rose \& Weinstein, 1988; Weinstein, 1986) and deal the solutions near the bifurcation point or they deal with a weaker notion of orbital stability (Cazenave \& Lions (1982), Cazenave (2003), Hajaiej \& Stuart (2004)). In a major contribution to the understanding of orbital stability, conditions were established in Grillakis et al. (1987) giving a rigorous setting for the Vakhitov-Kolokolov (V-K) criterion (1973) (Kivshar \& Sukhorukov, 2001). In our context, the V-K criterion states that $u_{\lambda}(x) \mathrm{e}^{-\mathrm{i} \lambda \tau}$ is orbital stable if

$$
\frac{\mathrm{d}}{\mathrm{d} \lambda} \int_{-\infty}^{\infty} u_{\lambda}(x)^{2} \mathrm{~d} x<0
$$

Under the hypotheses of Theorem 7.3, this was proved for all $\lambda \in(-\infty, \Lambda)$ in McLeod et al. (2003). In Stuart (2006), it is shown that the conditions introduced in Grillakis et al. (1987) to justify the V-K criterion are also satisfied. 


\section{REFERENCES}

Akhmanov, R.-V., Khorklov, R. V. \& Sukhorukov, A. P. (1972) Self-focusing, self-defocusing and self-modulation of laser beams. Laser Handbook (F. T. Arecchi \& E. O. Schulz Dubois eds). Amsterdam, the Netherlands: North-Holland, pp. 1152-1174.

Arcoya, D., Cingolani, S. \& GameZ, J. L. (1999) Asymmetric modes in symmetric non-linear optical wave guides. SIAM J. Math. Anal., 30, 1391-1400.

CAZEnave, T. (2003) Semilinear Schrödinger Equations. Courant Institute Lecture Notes No. 10. Providence, RI: AMS.

Cazenave, T. \& Lions, P. L. (1982) Orbital stability of standing waves for some non-linear Schrödinger equations. Commun. Math. Phys., 85, 549-561.

Grillakis, M., Shatah, J. \& Strauss, W. (1987) Stability theory of solitary waves in the presence of symmetry. J. Funct. Anal., 74, 160-197.

Hajaiej, H. \& Stuart, C. A. (2004) On the variational approach to the stability of standing waves for the non-linear Schrödinger equation. Adv. Nonlinear Stud., 4, 469-501.

JEANJEAN, H. \& STUART, C. A. (1999) Nonlinear eigenvalue problems having an unbounded branch of symmetric bound states. Adv. Differ. Equ., 4, 639-670.

Kivshar, Y. S. \& Sukhorukov, A. A. (2001) Stability of spatial optical solitons. Spatial Optical Solitons (T. Torruellas \& S. Trillo eds). New York: Springer, pp. 211-245.

Luther-Davies, B. \& Stegeman, G. I. (2001) Materials for spatial solitons. Spatial Solitons (S. Trillo \& W. Torruellas eds). Berlin: Springer, pp. 19-35.

Mcleod, J. B., Stuart, C. A. \& Troy, W. C. (2003) Stability of standing waves for some non-linear Schrödinger equations. Differ. Integr. Equ., 16, 1025-1038.

Rose, H. A. \& WeINSTEIN, M. I. (1988) On the bound states of the non-linear Schrödinger equation with a linear potential. Physica D, 30, 207-218.

Ruppen, H. J. (1997) Multiple TE-modes for planar self-focusing wave guides. Ann. Mat. Pura Appl., IV Ser., 172, 323-377.

SALEH, B. \& TeICH, M. (1991) Fundamentals of Photonics. New York: Wiley.

SMith, W. L. (1986) Nonlinear refractive index . Handbook of Laser Science and Technology, vol. 3 (M. J. Weber ed.). Boca Raton, FL: CRC Press.

Strauss, W. A. (1989) Nonlinear Wave Equations. CBMS Notes No. 73. Providence, RI: AMS

StUART, C. A. (1993) Guidance properties of planar waveguides. Arch. Rat. Mech. Anal., 125, 145-200.

STUART, C. A. (2004) Modelling axi-symmetric travelling waves in a dielectric with nonlinear refractive index. Milan J. Math., 72, 107-128.

STUART, C. A. (2006) Uniqueness and stability of ground states for some non-linear Schrödinger equations. J. Eur. Math. Soc., 18, 399-414.

Svelto, O. (1974) Self-focusing, self-trapping and self-phase modulation of laser beams . Progress in Optics, vol. 12 (E. Wolf ed.). Amsterdam, the Netherlands: North-Holland, pp. 3-50.

VAKhitov, M. G. \& Kolokolov, A. A. (1973) Stationary solutions of the wave equation in the medium with non-linearity saturation. Radiophys. Quantum Electron., 16, 783.

Weinstein, M. I. (1986) Lyapunov stability of ground states for non-linear dispersive evolution equations. Commun. Pure Appl. Math., 39, 51-68. 